\title{
Clinical Diagnosis and Management of Breast Cancer
}

\author{
Elizabeth S. McDonald ${ }^{1}$, Amy S. Clark ${ }^{2}$, Julia Tchou ${ }^{3}$, Paul Zhang ${ }^{4}$, and Gary M. Freedman ${ }^{5}$ \\ ${ }^{1}$ Breast Imaging Division, Department of Radiology, Perelman School of Medicine, University of Pennsylvania, Philadelphia, \\ Pennsylvania; ${ }^{2}$ Division of Hematology/Oncology, Department of Medicine, Perelman School of Medicine, University of \\ Pennsylvania, Philadelphia, Pennsylvania; ${ }^{3}$ Department of Surgery, Perelman School of Medicine, University of Pennsylvania, \\ Philadelphia, Pennsylvania; ${ }^{4}$ Department of Pathology, Perelman School of Medicine, University of Pennsylvania, Philadelphia, \\ Pennsylvania; and ${ }^{5}$ Department of Radiation Oncology, Perelman School of Medicine, University of Pennsylvania, Philadelphia, \\ Pennsylvania
}

\begin{abstract}
The diagnosis and management of breast cancer are undergoing a paradigm shift from a one-size-fits-all approach to an era of personalized medicine. Sophisticated diagnostics, including molecular imaging and genomic expression profiles, enable improved tumor characterization. These diagnostics, combined with newer surgical techniques and radiation therapies, result in a collaborative multidisciplinary approach to minimizing recurrence and reducing treatment-associated morbidity. This article reviews the diagnosis and treatment of breast cancer, including screening, staging, and multidisciplinary management.
\end{abstract}

Key Words: breast cancer diagnosis; breast cancer management; breast cancer imaging; targeted therapy; radiation; pathology; precision medicine

J Nucl Med 2016; 57:9S-16S

DOI: 10.2967/jnumed.115.157834

I

n this article, we address current approaches to breast cancer diagnosis and management. These approaches include screening recommendations; diagnostic imaging and pathologic assessment to determine the extent of disease; surgery and radiation treatment; and an array of systemic options, such as chemotherapy, endocrine therapy, and targeted agents (Fig. 1). We also consider the potential contribution of functional imaging to a new era of personalized, tumor-specific treatment.

\section{BREAST CANCER DIAGNOSIS}

\section{Screening}

Breast cancer is generally diagnosed through either screening or a symptom (e.g., pain or a palpable mass) that prompts a diagnostic exam. Screening of healthy women is associated with the detection of tumors that are smaller, have lower odds of metastasis, are more amenable to breast-conserving and limited axillary surgery, and are less likely to require chemotherapy (1). This scenario translates to reduced treatment-related morbidity and improved survival.

Received Aug. 25, 2015; revision accepted Oct. 21, 2015.

For correspondence or reprints contact: Elizabeth S. McDonald, Breast Imaging Division, Department of Radiology, University of Pennsylvania, Hospital of the University of Pennsylvania, 3400 Spruce St./1 Silverstein, Philadelphia, PA 19104-4283.

E-mail: elizabeth.mcdonald@uphs.upenn.edu

COPYRIGHT (C 2016 by the Society of Nuclear Medicine and Molecular Imaging, Inc.
The only screening modality proven to reduce breast cancerspecific mortality is mammography (2). Screening mammography leads to a $19 \%$ overall reduction in breast cancer mortality (3), with less benefit for women in their 40s (15\%) and more benefit for women in their 60s (32\%). As a result, screening mammography is recommended by the American Cancer Society beginning at age 45 , or sooner depending on individual preference. The potential negative aspects of screening mammography are falsepositive examinations, radiation exposure, pain, anxiety, and other negative psychologic effects. Mammography has a $61 \%$ chance of a false-positive result over a 10 -y period for women commencing screening between the ages of $40 \mathrm{y}$ and $50 \mathrm{y}$. The risk of a falsepositive examination decreases with older age (3). The US Preventative Task Force cited a $15 \%$ breast cancer-related mortality reduction for women who were $39-49$ y old and a mortality-related benefit from screening between ages 39 and 69 . However, the task force released a controversial report recommending only biennial screening mammography for women who were 50-74 y old, excluding younger women to a large extent because of the high rate of false-positive results (4). Mammography for women in the 39- to 49-y-old age group was recommended if indicated after the use of a risk-based model of breast cancer screening, such as the models developed by the Population-Based Research Optimizing Screening Through Personalized Regimens network (5), or if requested by a patient.

The addition of digital breast tomosynthesis to a conventional fullfield digital mammography examination reduces false-positive results and increases cancer detection (6). One concern about adding digital breast tomosynthesis to screening is the approximate doubling of the radiation dose over that of conventional full-field digital mammography alone (7). To address this issue, some institutions, such as ours, reconstruct synthetic 2-dimensional images from 3-dimensional tomosynthesis images; this process reduces the radiation dose by approximately $45 \%$ (7). Initial clinical experience with synthetic 2-dimensional images has demonstrated no increase in recall examinations, so that the most significant tomosynthesis benefit is maintained (8). Still, the reduction of false-positive examinations after tomosynthesis implementation has been modest $(16 / 1,000)(6)$, and practice changes such as a lower maximum acceptable recall rate of $9 \%-10 \%$ (currently it is $12 \%$ ) and an increase in the biopsy threshold from a $2 \%$ chance of malignancy to a $4 \%$ chance of malignancy (9) could have a greater impact on reducing harm from screening.

Supplementing mammography with other imaging modalities for higher-risk patients leads to the additional detection of mammographically occult cancers. A metaanalysis of 14 studies of high-risk women found that MRI had a higher sensitivity for malignancy 


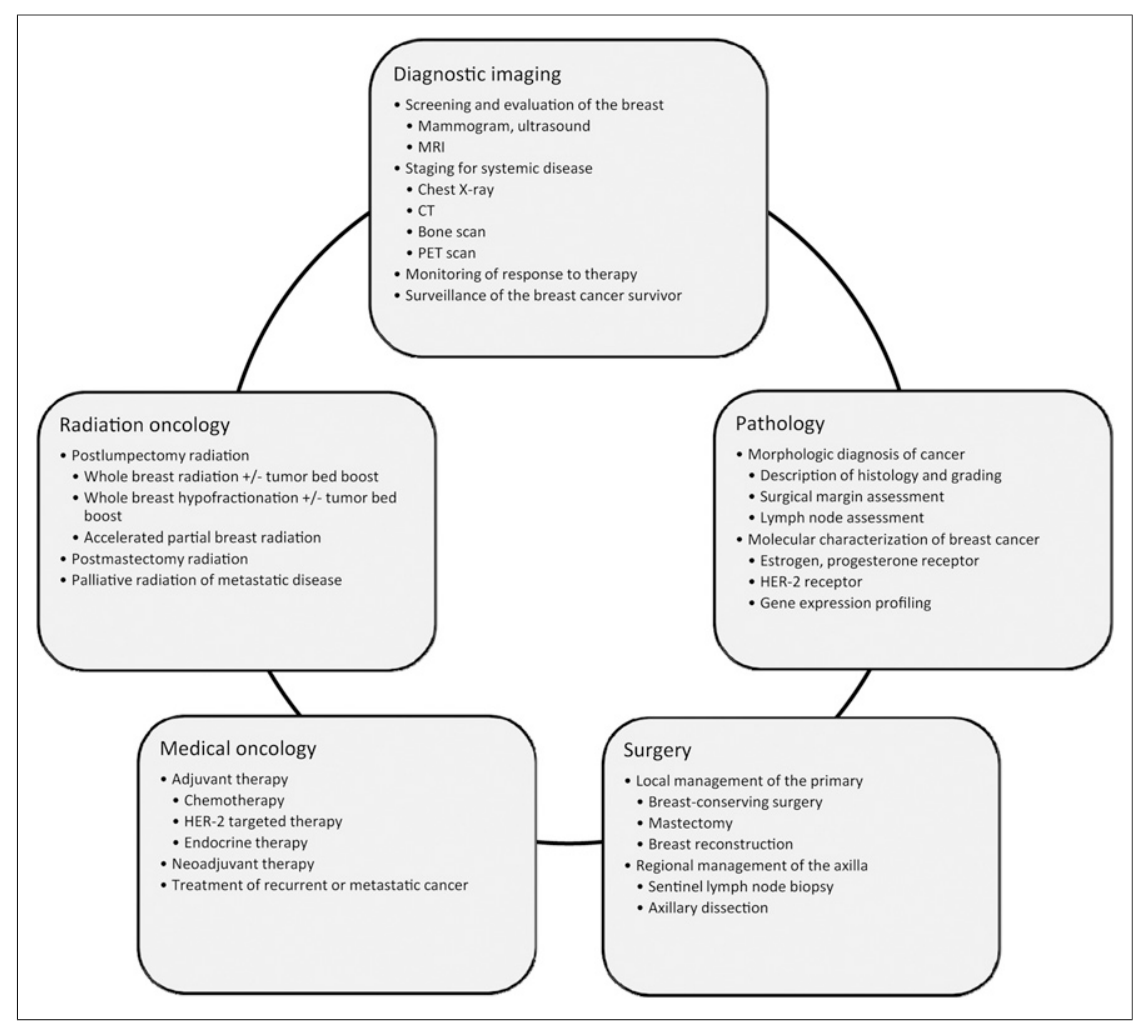

FIGURE 1. Overview of multidisciplinary breast cancer management.

$(84.6 \%)$ than mammography $(38.6 \%)$ or ultrasound $(39.6 \%)(10)$. Further, the use of MRI as an adjunct to mammography had a higher sensitivity for malignancy ( $92.7 \%$ ) than the use of ultrasound as an adjunct to mammography (52\%) (11). As a result, for women who have a lifetime risk of breast cancer of greater than $20 \%$, breast MRI as an adjunct to mammography is recommended by the American Cancer Society. This group includes women with genetic mutations that connote an increased risk of breast cancer and those with a history of radiation therapy for Hodgkin lymphoma that included the breast tissue. Ultrasound is a viable option for the screening of high-risk women who cannot have breast MRI or women with intermediate risk, such as those with dense breasts. The main limitations of screening ultrasound are a high rate of false-positive results and dependence on operator expertise (12). The high rate of false-positive results (and the low positive predictive value) of ultrasound has not yet met the minimum standard recommended by the Ultrasound Agency for Health Care Policy and Research (13). Concerning other screening modalities, some of which are discussed elsewhere in this supplement, the current American College of Radiology appropriateness criteria state, "There is insufficient evidence to support the use of [additional screening] imaging modalities such as thermography, breast-specific gamma imaging, positron emission mammography, and optical imaging" (14).

\section{Pathologic Evaluation}

Specimen Processing and Evaluation. In clinical practice, diseased tissue is usually obtained by fine-needle aspiration, core biopsy, or surgical excision. A diagnostic challenge for pathologists is the distinction of closely related diseases, such as atypical ductal hyperplasia and in situ disease, in situ disease and microinvasion, or ductal cancer and lobular cancer. Ancillary immunohistochemical and molecular tests can be used to assist the characterization of ambiguous morphology in many, but not all, cases. Features such as tissue handling, ischemic time, cautery, use of frozen sections, fixation, decalcification, and processing all are critical for the quality of the histologic sections used for microscopic evaluation and ancillary tests, such as immunohistochemistry (IHC), in situ hybridization, and molecular tests based on reverse transcription-polymerase chain reaction.

The size of the tumor is determined by careful clinical and pathologic correlation. When a breast cancer forms a distinct mass outward from a point of origin, the size can be easily assessed by imaging and gross pathologic examination. When a tumor arises in a poorly defined field of genetic instability and there is intratumoral normal tissue, accurate sizing can be challenging. In addition, finding and accurately measuring small cancers detected by advanced imaging can be difficult when they are not visible on gross inspection of the specimen, especially because the surgical specimen presented to the pathology laboratory might greatly deviate from the in vivo shape observed by the surgeon and radiologist due to breast tissue elasticity. Surgical specimens are typically marked with ink in 6 dimensions according to the orientation given by a surgeon. However, margin assessment after surgical resection is complicated by lack of marking

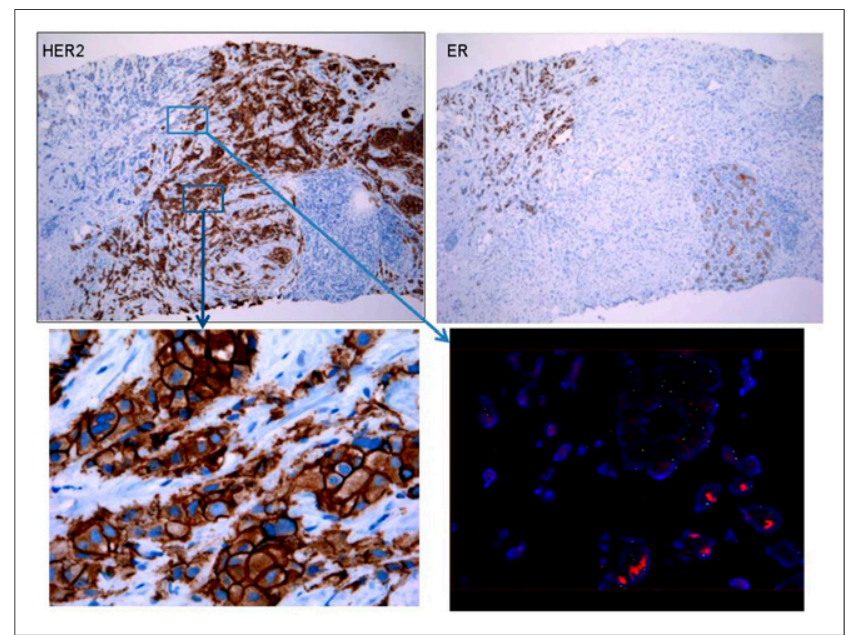

FIGURE 2. Invasive mammary cancer with heterogeneity of predictive marker staining. (Top left) IHC shows intense HER2 staining in some sections of tumor. (Top right) On adjacent section, IHC shows ER-negative staining of HER2-positive cells and ER-positive staining of HER2-negative cells. (Bottom left) Higher-power view of HER2 staining. (Bottom right) Fluorescence in situ hybridization for HER2 at interface of HER-positive and HER-negative cells also demonstrates signal heterogeneity. This patient was treated with chemotherapy and trastuzumab. Posttherapy histology demonstrated robust viable tumor that was exclusively HER2negative (not shown). 
standardization and potential artifacts from cautery or specimen handling.

Predictive Tumor Markers. Critical treatment decisions are made on the basis of protein expression assays that are independent of tumor morphologic characteristics. IHC analysis of paraffin sections is routinely performed for the evaluation of estrogen receptor (ER), progesterone receptor (PR), and Her-2/neu (HER2) status. Although widely used to predict responses to targeted agents, histologic tumor markers are limited by significant intratumoral variation, even within a single biopsy specimen (Fig. 2). RNA and DNA can also be tested in routine paraffin-embedded tissue samples, and in situ hybridization can detect HER2 amplification as a confirmatory test for IHC or as a stand-alone assay (Fig. 2, bottom right). There is great interest in other actionable targets in cancer genomes for precision therapy using next-generation gene sequencing (15).

DNA microarrays and high-throughput reverse transcriptionpolymerase chain reaction assays for multiple genes (e.g., 21 in Oncotype [Genomic Health, Inc.] and 70 in MammaPrint [Agendia]) can be used to categorize breast cancers into several prognostic groups (16). Gene assays are used to predict the risk of distant recurrence in early-stage breast cancer and to influence decisions about systemic therapy. These tests rely heavily on the assessment of ER and proliferation-related genes, such as Ki-67, and have largely replaced the use of other, single markers of risk in clinical practice. Still, as mentioned earlier, ER and PR expression can be heterogeneous and cellular proliferative status can also be variable within a single tumor (Fig. 3). Because any biopsy sample is subject to sampling error, and sectioning of the entire tumor for the analysis of predictive and prognostic biomarkers is not practical, imaging for breast cancer biomarkers can play a pivotal role in providing a global overview of gene expression. In addition to the markers already mentioned, other cancer biomarkers and oncogenic molecular genetic abnormalities have been reported (17); however, these are not yet widely accepted as the standard of care because of continued standardization of analyses, assay protocols, and analytic methodologies (18).

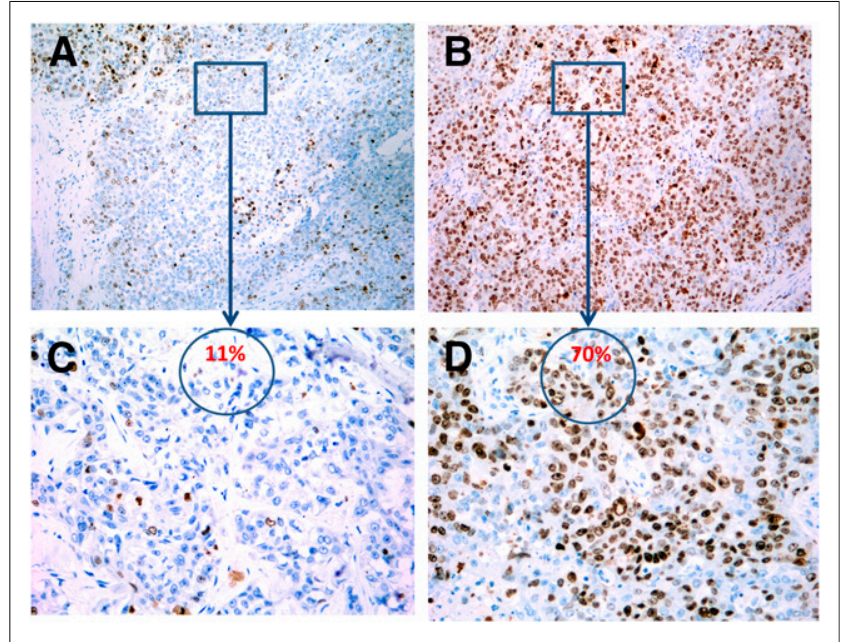

FIGURE 3. Triple-negative invasive breast cancer demonstrating variations in proliferative status. (A and B) Two low-power views of $\mathrm{Ki}-67$ IHC staining in single section from triple-negative tumor biopsy. (C and D) Higher-power (20x) views with calculation of representative $\mathrm{Ki}-67$ scores $(11 \%$ and $70 \%)$. Ki-67 scores were highly variable, even though these images were obtained from the same slide.

\section{Imaging and Staging}

Physical examination, mammography, or ultrasound for the diagnostic work-up of a patient with newly diagnosed breast cancer is usually sufficient for local-regional staging. MRI is sometimes recommended, especially when a patient is younger, a genetic mutation or multifocal disease is suspected, or a mammogram or ultrasound yields indeterminate findings. Although breast MRI does detect additional disease in the contralateral breast approximately $3 \%$ of the time (19), metaanalyses of preoperative breast MRI have shown an increase in rates of mastectomy (20) and no increase in local control after breast-conserving surgery (BCS) and radiation treatment (21). Studies of breast MRI have also shown a risk for overestimation of tumor size (22). Further, it is possible that small additional cancers detected by MRI would never be clinically significant or responsible for a local recurrence because of adjuvant systemic or whole-breast radiation treatments. MRI may play an important role in evaluating disease extent when more limited radiation to the tumor bed or only regional node irradiation is considered (23). In addition, breast MRI can be obtained in patients receiving neoadjuvant chemotherapy to assess responses and aid in surgical planning.

A chest radiograph and routine laboratory blood tests are sufficient for staging in a patient with clinical stage I or II breast cancer and no specific symptoms of metastatic disease. For suspected advanced (stage IIIB/C or IV) disease, National Comprehensive Cancer Network guidelines (version 1.2015) recommend either chest, abdomen, and pelvis CT or chest CT with abdomen and pelvis MRI as well as bone scan or sodium fluoride PET/CT. ${ }^{18} \mathrm{~F}-\mathrm{FDG}$ $\mathrm{PET} / \mathrm{CT}$ is listed as optional for assessing stage IIIB/C or IV disease but is not indicated for the staging of stage I or II disease. Supporting the use of PET to evaluate advanced breast cancer, a metaanalysis of 5 studies (547 patients) demonstrated a sensitivity for breast cancer of 0.97 (95\% confidence interval, 0.93-0.99) and a specificity of 0.95 (95\% confidence interval, 0.90-0.97) (24).

\section{BREAST CANCER TREATMENT}

\section{Surgery}

The primary means of local and regional breast cancer treatment remains surgical intervention. During the first half of the 20th century, women diagnosed with breast cancer were commonly treated by radical mastectomy, as first described by William Stewart Halsted in 1894. Breast conservation surgery (BCS) was pioneered by Fischer et al. (25) and Veronesi et al. (26), who reported that survival with lumpectomy and radiation was equivalent to that with mastectomy in the treatment of early breast cancer. Improved breast cancer screening resulted in diagnoses of nonpalpable cancers, necessitating the development of a localization approach for surgical treatment.

Breast-Conserving Approaches. Wire localization of a breast tumor is a mainstay of BCS. This procedure is routinely performed by a breast imaging radiologist on the day of surgery. Placement of the surgical incision on the breast is guided by cosmetic considerations and tumor location. A circumareolar location is ideal for a tumor 1-2 $\mathrm{cm}$ from the areolar margin, but when a tumor is more than $2 \mathrm{~cm}$ from the areola, an incision directly over the area of concern may be advantageous so that the lumpectomy site can be easily identified if a margin reexcision is necessary. After the initial incision is made, the length of the localization needle is noted, and dissection can take place directly along the needle track.

Radioactive seed localization reduces the time the patient spends in the hospital on the day of surgery and allows the surgeon to place the incision over the site with the highest counts without having to 
account for the site of entry of the needle, which may be in a quadrant different than the tumor. Studies comparing radioactive seed localization and wire localization demonstrated no significant difference in operative times and a possible lower reexcision rate with the seed localization technique (27).

In women with larger breasts, wide excision can be performed with an oncoplastic procedure, which usually involves breast reduction. This procedure was pioneered by Silverstein et al. for the surgical treatment of in situ breast cancer with the goals of obtaining surgical margins of more than $1 \mathrm{~cm}$ and avoiding wholebreast radiation (28). One caveat for this technique is that if there are positive margins, then reexcision may be difficult because of the rearrangement of tissue planes at the time of surgery. Therefore, patients should be counseled before choosing this option that complete mastectomy may be necessary if clean pathologic margins (no invasive breast cancer on ink or DCIS farther than $2 \mathrm{~mm}$ from ink) are not obtained.

Non-Breast-Conserving Approaches. For most women with screening-detected and early-stage breast cancer, mastectomy is a choice. However, mastectomy may be necessary for women who have had radiation to the affected side (for prior breast cancer or Hodgkin lymphoma) or for women with a relatively small breast in the setting of a large primary breast cancer, extensive calcifications, or multicentric disease. For women with a large primary breast cancer without extensive associated malignant calcifications, neoadjuvant chemotherapy may downstage the primary cancer and make breast conservation possible.

Most women electing mastectomy are candidates for immediate reconstruction (29). The surgical approach differs for women who do not elect reconstruction; a larger skin ellipse is removed. For women undergoing immediate reconstruction, skin-sparing mastectomy with or without preservation of the nipple may be performed. Nipple-sparing mastectomy is generally oncologically safe for in situ or stage I and II invasive cancers $(30,31)$. Some factors predicting nipple involvement are a tumor size of greater than $5 \mathrm{~cm}$, a distance from the tumor to the nipple of less than $2.5 \mathrm{~cm}$, negative ER and PR status, and positive HER2 status (32). Patients with malignant calcifications extending to within $2 \mathrm{~cm}$ of the nipple or inflammatory breast cancer are generally counseled against this procedure.

Axilla Staging Procedure. One of the major technical advances in breast surgery was the introduction of sentinel lymph node biopsy (SLNB) to replace the conventional axillary node dissection described by Giuliano et al. in 1994 (33). SLNB is associated with a significantly lower lymphedema risk $(<2 \%-3 \%)$ than complete axillary node dissection $(15 \%-20 \%)(34)$. The procedure is more than $98 \%$ accurate when results are negative, and further dissection is not needed. When SLNB results are positive, complete axillary dissection is not useful for improved local-regional control or survival in women who have no palpable adenopathy, 1 or 2 positive sentinel nodes, and no gross extranodal extension, as shown by a large randomized controlled clinical trial (American College of Surgeons Oncology Group Z0011 trial) (35).

Breast radiologists often perform fine-needle aspiration of nonpalpable axillary nodes with suspect imaging morphology. Given the results of the Z0011 trial, this approach poses a surgical dilemma in the management of the axilla because most of the involved patients would have been eligible for SLNB. Should these patients have full axillary dissection if the fine-needle aspiration results are positive, or should they still have SLNB if the results for the axilla are clinically negative? These questions warrant further investigation.
SLNB may be used in the clinically node-positive patient after a good response to neoadjuvant chemotherapy, with a few caveats. The ACOSOG Z1071 trial demonstrated that SLNB is feasible after neoadjuvant chemotherapy, with an overall rate of false-negative results of $12.6 \%$ (36). The rates of false-negative results decreased to $10.8 \%$ when both radiotracer and blue dye were used and to $9.1 \%$ when there were at least 3 sentinel nodes (36). In the SENTINA trial (37), one arm contained patients who converted from clinically positive to negative results for the axilla after neoadjuvant chemotherapy (arm C) and underwent SLNB and then complete axillary node dissection. The overall rate of false-negative results of SLNB was $14.2 \%$ when lymphatic mapping was performed by either radiocolloid or blue dye injection. However, subset analyses revealed that the rate of false-negative results was significantly lower when both radiocolloid and blue dye were used $(8.6 \%)$ and when at least 3 sentinel nodes were removed $(7.3 \%)$.

\section{Medical Oncology}

Several broad classes of drugs for treating breast cancer are available; tumor characteristics and disease extent determine the recommendation for systemic chemotherapy, endocrine therapy, or HER2-directed therapy. For early-stage breast cancer, these features are ER, PR, and HER2 status; lymph node involvement; and tumor size. For stage IV disease, the receptor status and the locations of metastatic sites are the main factors.

Chemotherapy. Adjuvant chemotherapy after definitive surgery is generally recommended for patients with disease at high risk of recurrence. The following clinicopathologic characteristics may be indications for chemotherapy: ER-, PR-, and HER2-negative; HER2positive; larger tumor size; and positive lymph nodes. For patients with negative results for lymph nodes and ER-positive tumors, RNAbased genomic testing can be used to better estimate the risk of a distant recurrence as well as to identify patients who will benefit most from chemotherapy (38). Genomic testing may also be considered for patients who have a limited number of positive lymph nodes after SNLB or axillary dissection to determine whether chemotherapy is indicated (39). For patients with high-risk disease, cytotoxic therapy should include both an anthracycline and a taxane. For lowrisk disease, anthracyclines are more commonly omitted. The decision to use chemotherapy should be based on a balance of the potential survival benefit with the patient's comorbidities and risk for complications.

HER2-Directed Therapy. For HER2-positive breast cancer, trastuzumab, a HER2-specific monoclonal antibody, improves the survival of patients with early-stage breast cancer and should be given in addition to chemotherapy (40). Because of the increased risk of heart failure with anthracycline- and trastuzumab-containing regimens, nonanthracycline, taxane-containing regimens can be used $(41,42)$. No trials have compared the various HER2 regimens; therefore, for patients with the highest risk, a standard regimen contains an anthracycline followed by a taxane with trastuzumab. No matter which chemotherapy is used, trastuzumab should be continued for 1 y (43), with cardiac monitoring every 3 mo. Clinicians can also consider adding pertuzumab, a monoclonal antibody that is directed against a different area on the HER2 receptor than trastuzumab. The results of NCT01358877, a phase III, double-blind, placebo-controlled trial in which patients with positive HER2 and lymph nodes were randomized to receive adjuvant pertuzumab or placebo along with standard adjuvant chemotherapy and trastuzumab, are pending. 
Endocrine Therapy. Patients with ER- or PR-positive breast cancer should receive endocrine therapy, such as an aromatase inhibitor. If there is concern about an increased risk of osteoporosis or aromatase inhibitor intolerance, tamoxifen can be prescribed. Until recently, tamoxifen was recommended for all premenopausal patients. However, the results of the randomized 3-arm SOFT trial suggested that ovarian suppression plus exemestane was superior to tamoxifen, but only in patients who received chemotherapy (44). Because chemotherapy was administered at the discretion of the treating physician, the benefit of ovarian suppression and exemestane was observed in patients with the highest risk of relapse (positive lymph nodes, large primary tumors, or HER2-positive disease). For premenopausal patients who do not receive chemotherapy, tamoxifen alone remains acceptable. Not surprisingly, tamoxifen alone is better tolerated than ovarian suppression plus exemestane, although only for the first $2 \mathrm{y}$. Endocrine therapy is recommended for at least $5 \mathrm{y}$; however, the results of the randomized ATLAS study indicated a $3 \%$ additional improvement in breast cancer mortality with $10 \mathrm{y}$ of tamoxifen rather than 5 (45).

Neoadjuvant Therapy. There are a variety of indications for neoadjuvant therapy: a tumor larger than $5 \mathrm{~cm}$ in a patient desiring breast conservation, a tumor fixed to the chest wall, locally advanced disease, and inflammatory breast cancer. Chemotherapy regimens are dependent on the receptor subtype; most will contain an anthracycline and a taxane. For HER2positive disease, trastuzumab and pertuzumab should be given concomitantly with the taxane $(46,47)$. For patients with locally advanced disease in whom chemotherapy will be too toxic but who still have curable breast cancer, neoadjuvant endocrine therapy can also be considered (48).

Therapy for Metastatic Disease. Because metastatic disease is not considered curable, the goal of therapy in the setting of metastatic disease is to extend life while minimizing symptoms or side effects. Patients with ER- or PR-positive and HER2-negative breast cancer usually receive endocrine therapy several times before being placed on single-agent chemotherapy. Recent data also support the addition of palbociclib, an oral inhibitor of cyclin-dependent kinases 4 and 6 , to first-line letrozole (49) and second-line fulvestrant (50) in patients with ER-positive metastatic disease. Patients with HER2-positive metastatic breast cancer should receive a taxane along with trastuzumab and pertuzumab as first-line therapy (51). Later therapies can include trastuzumab-emantine (52), lapatinib, or trastuzumab with other single-agent chemotherapeutics. The only class of agents available to patients with ER-, PR-, and HER2-negative breast cancer is chemotherapy.

Regardless of the treatment decision, the response to therapy in patients with metastatic disease should be assessed at predefined time intervals by clinical and imaging studies, including CT, bone scan, or PET/CT.

\section{Radiation}

Prospective randomized trials have confirmed that long-term mortality from breast cancer and overall patient survival are comparable for BCS plus radiation treatment and for mastectomy (53). BCS plus radiation treatment is also associated with very high local control rates (90\%-95\%) in the preserved breast within $10 \mathrm{y}$ from treatment; these rates are comparable to those obtained with mastectomy, with most women having a good or excellent cosmetic result $(54,55)$. The low rates of local recurrence in the modern era are due to progress in the multidisciplinary care of breast cancer: treatment of disease at an earlier stage because of detection by screening; improved imaging enabling appropriate patient selection for breast conservation; improved surgical techniques and margin pathology assessment; and improved radiation techniques, which may reduce marginal miss and radiation dose escalation, with a tumor bed boost, when indicated. Additionally, chemotherapy or endocrine systemic therapies, when indicated, are in widespread use, as discussed above. Radiation also has a proven role in the treatment of stage 0 breast cancer (ductal carcinoma in situ); 90\%-95\% long-term local control has been achieved with improved patient selection and surgical and radiation techniques $(56,57)$.

The past decade has seen considerable advances in the delivery of postoperative radiation that aim to optimize the treatment for each person's anatomy and reduce acute or long-term toxicity. Threedimensional planning with a CT simulator and either field-in-field 3-dimensional conformal radiation therapy (forward planning) or intensity-modulated radiation therapy (inverse planning) has replaced the simple 2-dimensional planned breast tangents. By reducing dose nonhomogeneity, these advances in techniques are associated with lower rates of complications, such as acute skin desquamation, edema, late fibrosis, or negative cosmetic effects on the breast $(58,59)$. In addition, techniques involving the prone position and deep-inspiration breath holding are now used for left-side breast cancer or larger breast size to reduce toxicity (particularly cardiac dose sparing) $(60,61)$.

Radiation After BCS. Randomized trials have confirmed that recurrence rates with $\mathrm{BCS}$ alone are higher than those with $\mathrm{BCS}$ plus radiation treatment, even in patients selected for favorable clinical and pathologic features (62-64). In addition, there is a small survival advantage of radiation in patients with invasive breast cancer. However, BCS without radiation may be an option for carefully selected women. In early-stage invasive breast cancer, the combination of older age, small tumor size, negative results for lymph nodes, and hormone sensitivity has been associated with a low risk for local recurrence after BCS without radiation (65). In ductal carcinoma in situ, BCS alone may be an option for tumors with a small size, wide margins, and a low to intermediate grade (66). With careful patient selection, subgroups of patients with these characteristics may have acceptably low local recurrence rates even without radiation (not likely to significantly affect the odds for survival) and a high probability for salvage therapy of local recurrences. Trials have not consistently required MRI staging, which could improve the detection of additional foci that are located away from the primary tumor bed and that could lead to early recurrences in patients not receiving radiation.

Regional Node Radiation. Radiation therapy has a role in the regional control of nodal disease in many patients with high-risk or node-positive stage II, and most patients with stage III, breast cancer. The primary area at risk for regional recurrences in patients with positive lymph nodes is the supraclavicular and high axillary region. Radiation is currently directed to these areas on the basis of pathology indications, such as the number of axillary nodes with positive results, the ratio of axillary nodes with positive results to those with negative results, the extent of axillary dissection, and extensive lymphovascular invasion. Even though PET/CT has a relatively low sensitivity for axillary disease $(60 \%)$, the specificity is very high (97\%) (67).

The role of elective radiation of the internal mammary chain remains controversial after $50 \mathrm{y}$ of study. Studies of elective nodal radiation have either yielded negative findings or resulted in only a 

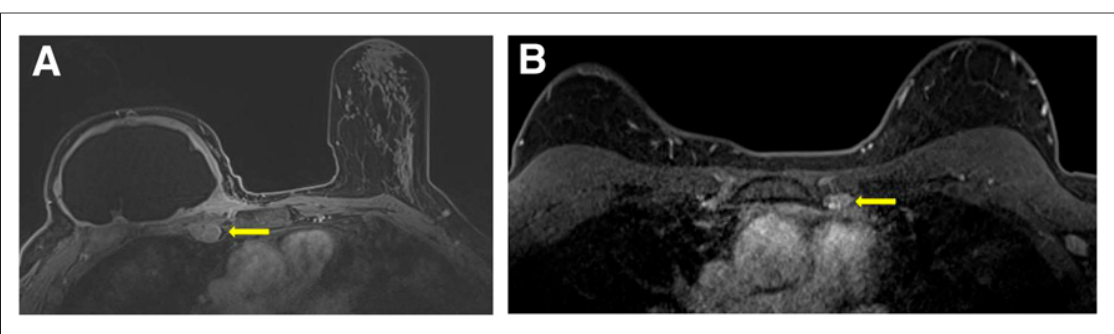

FIGURE 4. Internal mammary node enlargement detected by breast MRI in 2 patients (A and B). Imaging detected abnormal internal mammary lymph nodes (arrows) on basis of size and enhancement.

marginal disease-free survival benefit (67-69). Selection of patients for internal mammary node radiation is often recommended for subgroups at higher risk because of inner-quadrant tumor location with positive axillary nodes, extensive lymphovascular invasion, or hormone receptor-negative tumors. The negative findings in these trials of elective radiation may have been partly due to the randomization of large numbers of patients without actual disease involvement. Imaging could be used to select patients with nodal involvement before intervention (Fig.4), but this approach has not been used in prospective trials. PET/CT identifies occult lymph node metastases in the internal mammary chain in approximately $10 \%-15 \%$ of patients, leading to altered radiation field selection in $10 \%-16 \%$ of patients $(71,72)$.

Postmastectomy Radiation. In women treated by mastectomy, radiation is recommended for adjuvant treatment when there are clinical or pathologic factors predicting an intermediate to high risk ( $\geq 10 \%$ ) of local-regional recurrence (73). Randomized prospective trials have confirmed a reduction in local-regional recurrence and an improvement in survival with postmastectomy radiation in this subgroup of women (74). In contrast, women with a low risk for local-regional recurrence after mastectomy do not require radiation. Patients generally treated with postmastectomy radiation include those who have 4 or more positive axillary nodes, T3 tumor size, positive resection margins, and locally advanced or inflammatory breast cancer. Radiation is also recommended for patients who have 1-3 positive nodes and other risk factors for local-regional recurrence, such as lymphovascular invasion, young age, high-grade tumors, or hormone receptor-negative breast cancer (75).

Shortening Radiation Length. The past decade has seen advances in techniques for the delivery of postoperative radiation that aim to preserve high rates of local control but shorten overall treatment time, reduce cost, and improve convenience of care. Hypofractionation is the use of radiation treatments with fewer, larger doses than the conventional radiation fraction sizes of 1.8-2 Gy/d.

Hypofractionated whole-breast irradiation (WBI) has been firmly established as a standard of care for postlumpectomy radiation for early-stage breast cancer, in large part because of the favorable 10 -y results of 4 prospective randomized trials from Canada and the United Kingdom $(75,76)$. These trials showed equal 10-y local control as well as comparable or better cosmetic outcomes and late toxicities with hypofractionation. One of the issues regarding more widespread acceptance of WBI has been the relatively low accrual into the 4 major trials of certain subgroups of patients, such as those younger than $50 \mathrm{y}$ and requiring a boost or systemic chemotherapy. Currently, approximately $20 \%$ of women are treated with WBI (77). The Radiation Therapy Oncology Group completed a phase III randomized trial (RTOG
1005) of hypofractionated WBI with a concurrent boost that had the goals of expanding the use of hypofractionation by enrolling a patient population broader than that enrolled in the existing hypofractionated WBI studies and further reducing the treatment time to only $3 \mathrm{wk}$.

Accelerated partial breast irradiation (APBI) represents a departure from whole-breast irradiation because only the area around the primary tumor, including a small margin, is targeted with radiation. The major techniques used for APBI can be divided into external-beam radiation therapy and delivery of radiation through sources placed inside temporary internal catheters (brachytherapy) $(78,79)$. Because of the much smaller treatment volume, the radiation dose is increased and the treatment time is reduced, commonly twice a day for 10 fractions over 1 wk. Not all patients with early-stage breast cancer are suitable for APBI; in past trials with promising 5-y results, enrollment was generally limited to patients with small tumor sizes and favorable histologic characteristics. The degree to which young age or adverse pathologic features will influence local control with APBI is unknown. The National Surgical Adjuvant Breast and Bowel Project and the Radiation Therapy Oncology Group have completed a phase III randomized study (NSABP B-39/RTOG 0413) — one of many worldwide that are awaiting follow-up and publication of results - testing APBI against whole-breast radiation therapy, with endpoints of local control, survival, cosmetic outcome, and quality of life.

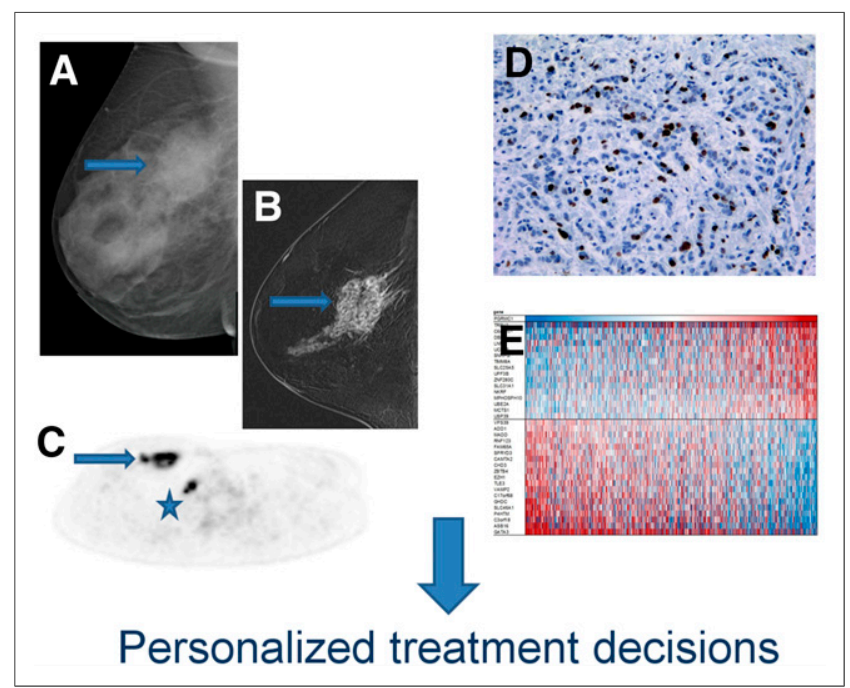

FIGURE 5. Precision medicine is the future of cancer therapy. Structural and functional information from imaging is combined with immunohistochemical and genomic information to make personalized treatment decisions. (A) Breast cancer (arrow) is largely obscured on mammography. (B) Variable uptake of gadolinium contrast agent on breast MRI (arrow) indicates heterogeneity of intratumoral blood flow. (C) Variable uptake of ${ }^{18}$ F-FDG PET radiotracer (arrow) indicates heterogeneity of glucose metabolism in primary breast cancer. Extraaxillary nodal disease is also demonstrated (*). (D) This information is combined with that from immunohistochemical assays, and mRNA expression (E) to determine a full tumor profile for treatment planning. 


\section{CONCLUSION}

Continued evolution of breast cancer diagnosis and management has resulted in a paradigm shift from standardized treatment regimens to "precision medicine" targeting the unique genetic compositions of patients and tumors. The treatment of breast cancer patients can be personalized by integrating analysis of standard immunohistochemical markers and gene expression with information from anatomic imaging as well as targeted functional imaging studies to tailor both treatment planning and response assessment (Fig. 5).

\section{DISCLOSURE}

This work was supported by the National Center for Research Resources and the National Center for Advancing Translational Sciences, National Institutes of Health, through grant UL1TR000003. The content is solely the responsibility of the authors and does not necessarily represent the official views of the NIH. This work was also supported by Komen Leadership grant AC140060 and Department of Energy training grant DE-SE0012476. No other potential conflict of interest relevant to this article was reported.

\section{REFERENCES}

1. Fuller MS, Lee CI, Elmore JG. Breast cancer screening: an evidence-based update. Med Clin North Am. 2015;99:451-468.

2. Berry DA, Cronin KA, Plevritis SK, et al. Effect of screening and adjuvant therapy on mortality from breast cancer. $N$ Engl J Med. 2005;353:1784-1792.

3. Pace LE, Keating NL. A systematic assessment of benefits and risks to guide breast cancer screening decisions. JAMA. 2014;311:1327-1335.

4. U.S. Preventive Services Task Force. Breast cancer: screening. http://www. uspreventiveservicestaskforce.org/Page/Topic/recommendation-summary/ breast-cancer-screening. Published November 2009. Accessed November 13, 2015.

5. Onega T, Beaber EF, Sprague BL, et al. Breast cancer screening in an era of personalized regimens: a conceptual model and National Cancer Institute initiative for risk-based and preference-based approaches at a population level. Cancer. 2014;120:2955-2964.

6. Friedewald SM, Rafferty EA, Rose SL, et al. Breast cancer screening using tomosynthesis in combination with digital mammography. JAMA. 2014;311:2499-2507.

7. Svahn TM, Houssami N, Sechopoulos I, Mattsson S. Review of radiation dose estimates in digital breast tomosynthesis relative to those in two-view full-field digital mammography. Breast. 2015;24:93-99.

8. Zuckerman SP, Conant EF, Weinstein S, Synnestvedt M, Korhonen K, McDonald ES. Impact on recall rates following implementation of synthesized 2D mammography in digital breast tomosynthesis screening. In: Radiologic Society of North America 2015 Meeting Proceedings; November 29-December 4, 2015; Chicago, IL.

9. Hall FM. Screening mammography guidelines: an alternative proactive approach. Radiology. 2014;273:646-651.

10. Lehman CD. Clinical indications: what is the evidence? Eur J Radiol. 2012;81 (suppl 1):S82-S84.

11. Berg WA. Tailored supplemental screening for breast cancer: what now and what next? AJR. 2009;192:390-399.

12. Berg WA, Blume JD, Cormack JB, et al. Combined screening with ultrasound and mammography vs mammography alone in women at elevated risk of breast cancer. JAMA. 2008;299:2151-2163.

13. Brem RF, Lenihan MJ, Lieberman J, Torrente J. Screening breast ultrasound: past, present, and future. AJR. 2015;204:234-240.

14. Mainiero MB, Lourenco A, Mahoney MC, et al. ACR Appropriateness Criteria Breast Cancer Screening. J Am Coll Radiol. 2013;10:11-14.

15. Bennett NC, Farah CS. Next-generation sequencing in clinical oncology: next steps towards clinical validation. Cancers (Basel). 2014;6:2296-2312.

16. Perou CM, Sorlie T, Eisen MB, et al. Molecular portraits of human breast tumours. Nature. 2000;406:747-752.

17. Ross JS, Linette GP, Stec J, et al. Breast cancer biomarkers and molecular medicine. Expert Rev Mol Diagn. 2003;3:573-585.

18. Allred DC, Harvey JM, Berardo M, Clark GM. Prognostic and predictive factors in breast cancer by immunohistochemical analysis. Mod Pathol. 1998;11:155-168.
19. Lehman CD, Gatsonis C, Kuhl CK, et al. MRI evaluation of the contralateral breast in women with recently diagnosed breast cancer. $N$ Engl J Med. 2007;356: 1295-1303.

20. Houssami N, Turner R, Morrow M. Preoperative magnetic resonance imaging in breast cancer: meta-analysis of surgical outcomes. Ann Surg. 2013;257:249-255.

21. Houssami N, Turner R, Macaskill P, et al. An individual person data meta-analysis of preoperative magnetic resonance imaging and breast cancer recurrence. J Clin Oncol. 2014;32:392-401.

22. Berg WA, Gutierrez L, NessAiver MS, et al. Diagnostic accuracy of mammography, clinical examination, US, and MR imaging in preoperative assessment of breast cancer. Radiology. 2004;233:830-849.

23. Dorn PL, Al-Hallaq HA, Haq F, et al. A prospective study of the utility of magnetic resonance imaging in determining candidacy for partial breast irradiation. Int J Radiat Oncol Biol Phys. 2013;85:615-622.

24. Xu G, Zhao L, He Z. Performance of whole-body PET/CT for the detection of distant malignancies in various cancers: a systematic review and metaanalysis. J Nucl Med. 2012;53:1847-1854.

25. Fischer JP, Wes AM, Tuggle CT, et al. Mastectomy with or without immediate implant reconstruction has similar 30-day perioperative outcomes. J Plast Reconstr Aesthet Surg. 2014;67:1515-1522.

26. Veronesi U, Saccozzi R, Del Vecchio M, et al. Comparing radical mastectomy with quadrantectomy, axillary dissection, and radiotherapy in patients with small cancers of the breast. N Engl J Med. 1981;305:6-11.

27. Gray RJ, Pockaj BA, Karstaedt PJ, Roarke MC. Radioactive seed localization of nonpalpable breast lesions is better than wire localization. Am J Surg. 2004;188: 377-380.

28. Silverstein MJ, Mai T, Savalia N, Vaince F, Guerra L. Oncoplastic breast conservation surgery: the new paradigm. J Surg Oncol. 2014;110:82-89.

29. Cordeiro PG. Breast reconstruction after surgery for breast cancer. $N$ Engl J Med. 2008;359:1590-1601.

30. De La Cruz L, Moody AM, Tappy EE, Blankenship SA, Hecht EM. Overall survival, disease-free survival, local recurrence, and nipple-areolar recurrence in the setting of nipple-sparing mastectomy: a meta-analysis and systematic review. Ann Surg Oncol. 2015;22:3241-3249.

31. Peled AW, Wang F, Foster RD, et al. Expanding the indications for total skinsparing mastectomy: is it safe for patients with locally advanced disease? Ann Surg Oncol. July 14, 2015 [Epub ahead of print].

32. Zhang H, Li Y, Moran MS, Haffty BG, Yang Q. Predictive factors of nipple involvement in breast cancer: a systematic review and meta-analysis. Breast Cancer Res Treat. 2015;151:239-249.

33. Giuliano AE, Kirgan DM, Guenther JM, Morton DL. Lymphatic mapping and sentinel lymphadenectomy for breast cancer. Ann Surg. 1994;220:391-398.

34. Lucci A, McCall LM, Beitsch PD, et al. Surgical complications associated with sentinel lymph node dissection (SLND) plus axillary lymph node dissection compared with SLND alone in the American College of Surgeons Oncology Group Trial Z0011. J Clin Oncol. 2007;25:3657-3663.

35. Giuliano AE, Hunt KK, Ballman KV, et al. Axillary dissection vs no axillary dissection in women with invasive breast cancer and sentinel node metastasis: a randomized clinical trial. JAMA. 2011;305:569-575.

36. Boughey JC, Suman VJ, Mittendorf EA, et al. Sentinel lymph node surgery after neoadjuvant chemotherapy in patients with node-positive breast cancer: the ACOSOG Z1071 (Alliance) clinical trial. JAMA. 2013;310:1455-1461.

37. Kuehn T, Bauerfeind I, Fehm T, et al. Sentinel-lymph-node biopsy in patients with breast cancer before and after neoadjuvant chemotherapy (SENTINA): a prospective, multicentre cohort study. Lancet Oncol. 2013;14:609-618.

38. Paik S, Shak S, Tang G, et al. A multigene assay to predict recurrence of tamoxifen-treated, node-negative breast cancer. $N$ Engl $\mathrm{J}$ Med. 2004;351:2817-2826.

39. Albain KS, Barlow WE, Shak S, et al. Prognostic and predictive value of the 21gene recurrence score assay in postmenopausal women with node-positive, oestrogen-receptor-positive breast cancer on chemotherapy: a retrospective analysis of a randomised trial. Lancet Oncol. 2010;11:55-65.

40. Perez EA, Romond EH, Suman VJ, et al. Trastuzumab plus adjuvant chemotherapy for human epidermal growth factor receptor 2-positive breast cancer: planned joint analysis of overall survival from NSABP B-31 and NCCTG N9831. J Clin Oncol. 2014;32:3744-3752.

41. Slamon D, Eiermann W, Robert N, et al. Adjuvant trastuzumab in HER2-positive breast cancer. $N$ Engl J Med. 2011;365:1273-1283.

42. Tolaney SM, Barry WT, Dang CT, et al. Adjuvant paclitaxel and trastuzumab for node-negative, HER2-positive breast cancer. N Engl J Med. 2015;372:134-141.

43. Mavroudis D, Saloustros E, Malamos N, et al. Six versus 12 months of adjuvant trastuzumab in combination with dose-dense chemotherapy for women with HER2-positive breast cancer: a multicenter randomized study by the Hellenic Oncology Research Group (HORG). Ann Oncol. 2015;26:1333-1340. 
44. Pagani O, Regan MM, Walley BA, et al. Adjuvant exemestane with ovarian suppression in premenopausal breast cancer. N Engl J Med. 2014;371:107-118.

45. Davies C, Pan H, Godwin J, et al. Long-term effects of continuing adjuvant tamoxifen to 10 years versus stopping at 5 years after diagnosis of oestrogen receptorpositive breast cancer: ATLAS, a randomised trial. Lancet. 2013;381:805-816.

46. Gianni L, Pienkowski T, Im YH, et al. Efficacy and safety of neoadjuvant pertuzumab and trastuzumab in women with locally advanced, inflammatory, or early HER2-positive breast cancer (NeoSphere): a randomised multicentre, open-label, phase 2 trial. Lancet Oncol. 2012;13:25-32.

47. Schneeweiss A, Chia S, Hickish T, et al. Pertuzumab plus trastuzumab in combination with standard neoadjuvant anthracycline-containing and anthracyclinefree chemotherapy regimens in patients with HER2-positive early breast cancer: a randomized phase II cardiac safety study (TRYPHAENA). Ann Oncol. 2013;24: 2278-2284.

48. Leal F, Liutti VT, Antunes Dos Santos VC, et al. Neoadjuvant endocrine therapy for resectable breast cancer: a systematic review and meta-analysis. Breast. 2015; 24:406-412.

49. Finn RS, Crown JP, Lang I, et al. The cyclin-dependent kinase 4/6 inhibitor palbociclib in combination with letrozole versus letrozole alone as first-line treatment of oestrogen receptor-positive, HER2-negative, advanced breast cancer (PALOMA-1/TRIO-18): a randomised phase 2 study. Lancet Oncol. 2015;16: 25-35.

50. Turner NC, Ro J, André F, et al. Palbociclib in hormone-receptor-positive advanced breast cancer. N Engl J Med. 2015;373:209-219.

51. Swain SM, Baselga J, Kim SB, et al. Pertuzumab, trastuzumab, and docetaxel in HER2-positive metastatic breast cancer. N Engl J Med. 2015;372:724-734.

52. Krop IE, Kim SB, González-Martin A, et al. Trastuzumab emtansine versus treatment of physician's choice for pretreated HER2-positive advanced breast cancer (TH3RESA): a randomised, open-label, phase 3 trial. Lancet Oncol. 2014;15: 689-699.

53. Jatoi I, Proschan MA. Randomized trials of breast-conserving therapy versus mastectomy for primary breast cancer: a pooled analysis of updated results. Am J Clin Oncol. 2005;28:289-294.

54. Anderson SJ, Wapnir I, Dignam JJ, et al. Prognosis after ipsilateral breast tumor recurrence and locoregional recurrences in patients treated by breast-conserving therapy in five National Surgical Adjuvant Breast and Bowel Project protocols of node-negative breast cancer. J Clin Oncol. 2009;27:2466-2473.

55. Haviland JS, A'Hern R, Bentzen SM, Whelan T, Bliss JM. Radiotherapy for breast cancer, the TARGIT-A trial. Lancet. 2014;383:1716-1717.

56. Turaka A, Freedman GM, Li T, et al. Young age is not associated with increased local recurrence for DCIS treated by breast-conserving surgery and radiation. $J$ Surg Oncol. 2009;100:25-31.

57. Whaley JT, Lester-Coll NH, Morrissey SM, Milby AB, Hwang WT, Prosnitz RG. Use of postexcision preirradiation mammography in patients with ductal carcinoma in situ of the breast treated with breast-conserving therapy. Pract Radiat Oncol. 2013;3:e107-e112.

58. Freedman GM, Li T, Nicolaou N, Anderson P. Breast intensity modulated radiation therapy reduces time spent with acute dermatitis for women of all breast sizes during radiation. Int J Radiat Oncol Biol Phys. 2009;74:689-694.

59. Keller LM, Sopka DM, Li T, et al. Five-year results of whole breast intensity modulated radiation therapy for the treatment of early stage breast cancer: the Fox Chase Cancer Center experience. Int J Radiat Oncol Biol Phys. 2012;84: 881-887.

60. Formenti SC, DeWyngaert JK, Jozsef G, Goldberg JD. Prone vs supine positioning for breast cancer radiotherapy. JAMA. 2012;308:861-863.
61. Eldredge-Hindy H, Lockamy V, Crawford A, et al. Active Breathing Coordinator reduces radiation dose to the heart and preserves local control in patients with left breast cancer: report of a prospective trial. Pract Radiat Oncol. 2015; 5:4-10.

62. Correa C, McGale P, Taylor C, et al. Overview of the randomized trials of radiotherapy in ductal carcinoma in situ of the breast. J Natl Cancer Inst Monogr. 2010;2010:162-177.

63. McCormick B, Winter K, Hudis C, et al. RTOG 9804: a prospective randomized trial for good-risk ductal carcinoma in situ comparing radiotherapy with observation. J Clin Oncol. 2015;33:709-715.

64. Darby S, McGale P, Correa C, et al. Effect of radiotherapy after breast-conserving surgery on 10-year recurrence and 15-year breast cancer death: meta-analysis of individual patient data for 10,801 women in 17 randomised trials. Lancet. 2011;378: 1707-1716.

65. Hughes KS, Schnaper LA, Bellon JR, et al. Lumpectomy plus tamoxifen with or without irradiation in women age 70 years or older with early breast cancer: long-term follow-up of CALGB 9343. J Clin Oncol. 2013;31:2382-2387.

66. Hughes LL, Wang M, Page DL, et al. Local excision alone without irradiation for ductal carcinoma in situ of the breast: a trial of the Eastern Cooperative Oncology Group. J Clin Oncol. 2009;27:5319-5324.

67. Robertson IJ, Hand F, Kell MR. FDG-PET/CT in the staging of local/regional metastases in breast cancer. Breast. 2011;20:491-494.

68. Hennequin C, Bossard N, Servagi-Vernat S, et al. Ten-year survival results of a randomized trial of irradiation of internal mammary nodes after mastectomy. Int J Radiat Oncol Biol Phys. 2013;86:860-866.

69. Poortmans PM, Collette S, Kirkove C, et al. Internal mammary and medial supraclavicular irradiation in breast cancer. N Engl J Med. 2015;373:317-327.

70. Whelan TJ, Olivotto IA, Parulekar WR, et al. Regional nodal irradiation in earlystage breast cancer. N Engl J Med. 2015;373:307-316.

71. Koolen BB, Valdes Olmos RA, Elkhuizen PH, et al. Locoregional lymph node involvement on ${ }^{18} \mathrm{~F}$-FDG PET/CT in breast cancer patients scheduled for neoadjuvant chemotherapy. Breast Cancer Res Treat. 2012;135:231-240.

72. Ng SP, David S, Alamgeer M, Ganju V. Impact of pretreatment combined ${ }^{18} \mathrm{~F}-$ fluorodeoxyglucose positron emission tomography/computed tomography staging on radiation therapy treatment decisions in locally advanced breast cancer. Int J Radiat Oncol Biol Phys. 2015;93:111-117.

73. Gradishar WJ, Anderson BO, Blair SL, et al. Breast cancer version 3.2014. J Natl Compr Canc Netw. 2014;12:542-590.

74. McGale P, Taylor C, Correa C, et al. Effect of radiotherapy after mastectomy and axillary surgery on 10-year recurrence and 20-year breast cancer mortality: metaanalysis of individual patient data for 8135 women in 22 randomised trials. Lancet. 2014;383:2127-2135.

75. Haviland JS, Owen JR, Dewar JA, et al. The UK Standardisation of Breast Radiotherapy (START) trials of radiotherapy hypofractionation for treatment of early breast cancer: 10-year follow-up results of two randomised controlled trials. Lancet Oncol. 2013;14:1086-1094.

76. Whelan TJ, Pignol J-P, Levine MN, et al. Long-term results of hypofractionated radiation therapy for breast cancer. $N$ Engl J Med. 2010;362:513-520.

77. Bekelman JE, Sylwestrzak G, Barron J, et al. Uptake and costs of hypofractionated vs conventional whole breast irradiation after breast conserving surgery in the United States, 2008-2013. JAMA. 2014;312:2542-2550.

78. Arthur DW, Vicini FA. Accelerated partial breast irradiation as a part of breast conservation therapy. J Clin Oncol. 2005;23:1726-1735.

79. Beitsch PD, Shaitelman SF, Vicini FA. Accelerated partial breast irradiation. $J$ Surg Oncol. 2011;103:362-368. 